\title{
Asymptotics of Eigenvalues for Regular Sturm-Liouville Problems with Spectral Parameter-Dependent Boundary Conditions and Symmetric Single Well Potential
}

\author{
ELIF BAŞKAYA (iD
}

Department of Mathematics, Faculty of Science, Karadeniz, Technical University, 61080, Trabzon, Turkey.

\author{
Received: 01-01-2021 • Accepted: 14-02-2021
}

\begin{abstract}
Aвstract. In this study, we find asymptotic estimates of eigenvalues for regular Sturm-Liouville problems having the eigenvalue parameter in all boundary conditions with the symmetric single well potential that is symmetric to the midpoint of the related interval and nonincreasing on the first semi-region of the related interval.
\end{abstract}

2010 AMS Classification: 34L10

Keywords: Sturm-Liouville problem, symmetric single well potential, eigenvalue parameter in the boundary conditions.

\section{INTRODUCTION}

In this paper, we consider the boundary value problem

$$
\begin{aligned}
y^{\prime \prime}(t)+\{\lambda-q(t)\} y(t) & =0, \quad t \in[0, a], \\
a_{1} y(0)+a_{2} y^{\prime}(0) & =\lambda\left[a_{1}^{\prime} y(0)+a_{2}^{\prime} y^{\prime}(0)\right], \\
b_{1} y(a)+b_{2} y^{\prime}(a) & =\lambda\left[b_{1}^{\prime} y(a)+b_{2}^{\prime} y^{\prime}(a)\right],
\end{aligned}
$$

where $\lambda$ is a real parameter, $q(t)$ is a real-valued, continuous function and $a_{i}, b_{i}, a_{i}^{\prime}, b_{i}^{\prime}$ for $i=1,2$ are real constants. We remark that this problem (1.1)-(1.3) is different from the usual regular Sturm-Liouville problem because spectral parameter $\lambda$ is seen in the boundary conditions. Such problems often arise from physical problems, for example, vibration of a string, quantum mechanics and geophysics. Fulton gives more than a hundred references about these problems in [15] and [16] (see also [22]) so his works serve as a historical guide. There are a lot of studies with SturmLiouville problems that the spectral parameter appears in the boundary conditions, some of them are [9-13], [17-20].

Besides, Equation (1.1) is equal to one-dimensional Schrödinger equation and especially in recent years, since quantum mechanic has gained importance, there are a lot of studies on eigenvalues of Hill's equation and Schrödinger's operator with symmetric single well potential such as anharmonic oscillator. The eigenvalues of these equations represent excitation energy and eigenfunctions are named as wavefunction in physics. A symmetric single well potential on $[0, a]$ is defined as symmetric with respect to the midpoint $a / 2$ and nonincreasing on $[0, a / 2]$. For example, in a symmetric hydrogen bond that occurs in many biological structures such as DNA and water, the proton free energy landscape is a symmetric single well potential. The unusual case of very strong H-bonds, features symmetric single-well proton

Email address: elifbekar@ktu.edu.tr (E. Başkaya) 
free energy profiles. In this particular case the energy minimum corresponds to the proton centered in the middle of the H-bond and therefore no PT transfer barrier exits [28]. Stationary states for a particle moving in a single-well, steeper than parabolic, potential driven by Lévy noise can be bimodal. [5] and [8] have verified that the stationary states in symmetric single-well potentials can be characterized by more than two modal values. [27] discusses analytical and numerical results for nonharmonic, undamped, single-well, stochastic oscillators driven by additive noises. It focuses on average kinetic, potential, and total energies together with the corresponding distributions under random drivings, involving Gaussian white, Ornstein-Uhlenbeck, and Markovian dichotomous noises. In [6], they study the inverse nodal problem and the eigenvalue gap for the one-dimensional sloshing problem with the $\mathrm{p}$-Laplacian operator. They investigate the eigenvalue gap under the restriction of symmetric single-well depth functions. The others of the eigenvalue problems with symmetric single well potential can be found in [1-3], [7], [14], [23-26].

In this study, we gain asymptotic approximations for eigenvalues $\lambda_{n}$ of (1.1)-(1.3) with symmetric single well potential q. We note that a symmetric single well potential on $[0, a]$ means that a continuous function $q(t)$ is symmetric on $[0, a]$ and non-increasing on $\left[0, \frac{a}{2}\right]$, so we have $q(t)=q(a-t)$ mathematically. We assume without loss of generality that $q(t)$ has a mean value zero.

\section{The Method}

At the beginning of our method, we should remark that $q^{\prime}(t)$ exists since a monotone function on an interval I is differentiable almost everywhere on I [21].

Our method is based on [4]. If we rearrange its main theorems for $a=0$ and $b=a$, we get the following results:

Theorem 2.1. The eigenvalues $\lambda_{n}$ of (1.1)-(1.3) satisfy as $n \rightarrow \infty$,

i) if $a_{2}^{\prime} \neq 0$ and $b_{2}^{\prime} \neq 0$,

$$
\begin{aligned}
\lambda_{n}^{1 / 2} & =\frac{(n+1) \pi}{a}+\frac{1}{(n+1) \pi}\left\{\frac{a_{2}^{\prime} b_{1}^{\prime}-a_{1}^{\prime} b_{2}^{\prime}}{a_{2}^{\prime} b_{2}^{\prime}}-\frac{a}{4(n+1) \pi} \int_{0}^{a} q^{\prime}(x)\left(\sin \frac{2(n+1) \pi x}{a}\right) d x\right. \\
& -\frac{a^{2}}{(n+1)^{2} \pi^{2}}\left[\frac{-3 b_{1}^{\prime} b_{2}^{\prime} b_{2}+3 b_{1}\left(b_{2}^{\prime}\right)^{2}+\left(b_{1}^{\prime}\right)^{3}}{3\left(b_{2}^{\prime}\right)^{3}}\right] \\
& +\frac{a^{2}}{(n+1)^{2} \pi^{2}}\left[\frac{-3 a_{1}^{\prime} a_{2}^{\prime} a_{2}+3 a_{1}\left(a_{2}^{\prime}\right)^{2}+\left(a_{1}^{\prime}\right)^{3}}{3\left(a_{2}^{\prime}\right)^{3}}\right]+\frac{a^{2}}{2(n+1)^{2} \pi^{2}} \frac{a_{1}^{\prime}}{a_{2}^{\prime}}[q(a)-q(0)] \\
& \left.-\frac{a^{2}}{2(n+1)^{2} \pi^{2}} \frac{a_{1}^{\prime}}{a_{2}^{\prime}} \int_{0}^{a} q^{\prime}(x)\left(\cos \frac{2(n+1) \pi x}{a}\right) d x\right\}+O\left(n^{-4} \eta(n)\right)+O\left(n^{-3} \eta^{2}(n)\right),
\end{aligned}
$$

ii) if $a_{2}^{\prime} \neq 0$ and $b_{2}^{\prime}=0$,

$$
\begin{aligned}
\lambda_{n}^{1 / 2} & =\frac{(2 n+3) \pi}{2 a}+\frac{2}{(2 n+3) \pi}\left\{\frac{a_{2}^{\prime} b_{2}-a_{1}^{\prime} b_{1}^{\prime}}{a_{2}^{\prime} b_{1}^{\prime}}-\frac{a}{2(2 n+3) \pi} \int_{0}^{a} q^{\prime}(x)\left(\sin \frac{(2 n+3) \pi x}{a}\right) d x\right. \\
& +\frac{4 a^{2}}{(2 n+3)^{2} \pi^{2}}\left[\frac{3 b_{1}^{\prime} b_{1} b_{2}-b_{2}^{3}}{3\left(b_{1}^{\prime}\right)^{3}}\right]+\frac{4 a^{2}}{(2 n+3)^{2} \pi^{2}}\left[\frac{-3 a_{1}^{\prime} a_{2}^{\prime} a_{2}+3 a_{1}\left(a_{2}^{\prime}\right)^{2}+\left(a_{1}^{\prime}\right)^{3}}{3\left(a_{2}^{\prime}\right)^{3}}\right] \\
& -\frac{2 a^{2}}{(2 n+3)^{2} \pi^{2}} \frac{a_{1}^{\prime}}{a_{2}^{\prime}}[q(a)+q(0)] \\
& \left.-\frac{2 a^{2}}{(2 n+3)^{2} \pi^{2}} \frac{a_{1}^{\prime}}{a_{2}^{\prime}} \int_{0}^{a} q^{\prime}(x)\left(\cos \frac{(2 n+3) \pi x}{a}\right) d x\right\}+O\left(n^{-4} \eta(n)\right)+O\left(n^{-3} \eta^{2}(n)\right),
\end{aligned}
$$

where $\eta(\lambda):=\sup _{0 \leq t \leq a} F(t, \lambda)$ so that 


$$
F(t, \lambda):=\left\{\begin{array}{lr}
\left|\int_{t}^{a} e^{2 i \lambda^{1 / 2} x} q^{\prime}(x) d x\right| / \int_{t}^{a}\left|q^{\prime}(x)\right| d x, & \text { if } \int_{t}^{a}\left|q^{\prime}(x)\right| d x \neq 0, \\
0 & , \text { if } \int_{t}^{a}\left|q^{\prime}(x)\right| d x=0 .
\end{array}\right.
$$

Theorem 2.2. The eigenvalues $\lambda_{n}$ of (1.1)-(1.3) satisfy as $n \rightarrow \infty$,

i) if $a_{2}^{\prime}=0$ and $b_{2}^{\prime} \neq 0$,

$$
\begin{aligned}
\lambda_{n}^{1 / 2} & =\frac{(2 n+3) \pi}{2 a}+\frac{2}{(2 n+3) \pi}\left\{\frac{a_{1}^{\prime} b_{1}^{\prime}-a_{2} b_{2}^{\prime}}{a_{1}^{\prime} b_{2}^{\prime}}-\frac{a}{2(2 n+3) \pi} \int_{0}^{a} q^{\prime}(x)\left(\sin \frac{(2 n+3) \pi x}{a}\right) d x\right. \\
& -\frac{4 a^{2}}{(2 n+3)^{2} \pi^{2}}\left[\frac{3 b_{1}\left(b_{2}^{\prime}\right)^{2}-3 b_{1}^{\prime} b_{2}^{\prime} b_{2}+\left(b_{1}^{\prime}\right)^{3}}{3\left(b_{2}^{\prime}\right)^{3}}\right] \\
& +\frac{4 a^{2}}{(2 n+3)^{2} \pi^{2}}\left[\frac{-3 a_{1}^{\prime} a_{1} a_{2}+a_{2}^{3}}{3\left(a_{1}^{\prime}\right)^{3}}\right]+\frac{2 a^{2}}{(2 n+3)^{2} \pi^{2}} \frac{a_{2}}{a_{1}^{\prime}}[q(a)+q(0)] \\
& \left.+\frac{2 a^{2}}{(2 n+3)^{2} \pi^{2}} \frac{a_{2}}{a_{1}^{\prime}} \int_{0}^{a} q^{\prime}(x)\left(\cos \frac{(2 n+3) \pi x}{a}\right) d x\right\}+O\left(n^{-4} \eta(n)\right)+O\left(n^{-3} \eta^{2}(n)\right),
\end{aligned}
$$

ii) if $a_{2}^{\prime}=0$ and $b_{2}^{\prime}=0$,

$$
\begin{aligned}
\lambda_{n}^{1 / 2} & =\frac{(n+2) \pi}{a}+\frac{1}{(n+2) \pi}\left\{\frac{a_{1}^{\prime} b_{2}-a_{2} b_{1}^{\prime}}{a_{1}^{\prime} b_{1}^{\prime}}+\frac{a}{4(n+2) \pi} \int_{0}^{a} q^{\prime}(x)\left(\sin \frac{2(n+2) \pi x}{a}\right) d x\right. \\
& -\frac{a^{2}}{(n+2)^{2} \pi^{2}}\left[\frac{3 b_{1}^{\prime} b_{1} b_{2}-b_{2}^{3}}{3\left(b_{1}^{\prime}\right)^{3}}\right]+\frac{a^{2}}{(n+2)^{2} \pi^{2}}\left[\frac{-3 a_{1}^{\prime} a_{1} a_{2}+a_{2}^{3}}{3\left(a_{1}^{\prime}\right)^{3}}\right] \\
& -\frac{a^{2}}{(n+2)^{2} \pi^{2}} \frac{a_{2}}{2 a_{1}^{\prime}}[q(a)-q(0)] \\
& \left.+\frac{a^{2}}{(n+2)^{2} \pi^{2}} \frac{a_{2}}{2 a_{1}^{\prime}} \int_{0}^{a} q^{\prime}(x)\left(\cos \frac{2(n+2) \pi x}{a}\right) d x\right\}+O\left(n^{-4} \eta(n)\right)+O\left(n^{-3} \eta^{2}(n)\right) .
\end{aligned}
$$

\section{The Main Results}

To obtain asymptotics approximations for eigenvalues $\lambda_{n}$ of (1.1)-(1.3) with symmetric single well potential q, we calculate integral terms of Theorem 2.1 and Theorem 2.2. Therefore, we first compute the following integrals:

Lemma 3.1. If $q(t)$ is a symmetric single well potential, then

i)

ii)

$$
\int_{0}^{a} q^{\prime}(x) \sin \left(\frac{2(n+1) \pi x}{a}\right) d x=2 \int_{0}^{a / 2} q^{\prime}(x) \sin \left(\frac{2(n+1) \pi x}{a}\right) d x,
$$

$$
\int_{0}^{a} q^{\prime}(x) \sin \left(\frac{(2 n+3) \pi x}{a}\right) d x=2 \int_{0}^{a / 2} q^{\prime}(x) \sin \left(\frac{(2 n+3) \pi x}{a}\right) d x
$$

iii)

iv)

$$
\int_{0}^{a} q^{\prime}(x) \sin \left(\frac{2(n+2) \pi x}{a}\right) d x=2 \int_{0}^{a / 2} q^{\prime}(x) \sin \left(\frac{2(n+2) \pi x}{a}\right) d x,
$$

$$
\int_{0}^{a} q^{\prime}(x) \cos \left(\frac{2(n+1) \pi x}{a}\right) d x=0
$$


v)

vi)

$$
\int_{0}^{a} q^{\prime}(x) \cos \left(\frac{(2 n+3) \pi x}{a}\right) d x=0
$$

$$
\int_{0}^{a} q^{\prime}(x) \cos \left(\frac{2(n+2) \pi x}{a}\right) d x=0 .
$$

Proof. i)

$$
\begin{aligned}
\int_{0}^{a} q^{\prime}(x) \sin \left(\frac{2(n+1) \pi x}{a}\right) d x & =\int_{0}^{a / 2} q^{\prime}(x) \sin \left(\frac{2(n+1) \pi x}{a}\right) d x+\int_{a / 2}^{a} q^{\prime}(x) \sin \left(\frac{2(n+1) \pi x}{a}\right) d x \\
& =\int_{0}^{a / 2} q^{\prime}(x) \sin \left(\frac{2(n+1) \pi x}{a}\right) d x-\int_{a / 2}^{a} q^{\prime}(a-x) \sin \left(\frac{2(n+1) \pi x}{a}\right) d x \\
& =\int_{0}^{a / 2} q^{\prime}(x) \sin \left(\frac{2(n+1) \pi x}{a}\right) d x+\int_{a / 2}^{0} q^{\prime}(u) \sin \left(\frac{2(n+1) \pi(a-u)}{a}\right) d u \\
& =\int_{0}^{a / 2} q^{\prime}(x) \sin \left(\frac{2(n+1) \pi x}{a}\right) d x+\int_{0}^{a / 2} q^{\prime}(u) \sin \left(\frac{2(n+1) \pi u}{a}\right) d u \\
& =2 \int_{0}^{a / 2} q^{\prime}(x) \sin \left(\frac{2(n+1) \pi x}{a}\right) d x
\end{aligned}
$$

The second equality holds since $q(t)$ is symmetric and $q^{\prime}(t)$ exists, so $q^{\prime}(t)=-q^{\prime}(a-t)$.

ii) and iii) can be proved similarly to i).

iv) Because of $q^{\prime}(t)=-q^{\prime}(a-t)$, we have

$$
\begin{aligned}
\int_{0}^{a} q^{\prime}(x) \cos \left(\frac{2(n+1) \pi x}{a}\right) d x & =\int_{0}^{a / 2} q^{\prime}(x) \cos \left(\frac{2(n+1) \pi x}{a}\right) d x+\int_{a / 2}^{a} q^{\prime}(x) \cos \left(\frac{2(n+1) \pi x}{a}\right) d x \\
& =\int_{0}^{a / 2} q^{\prime}(x) \cos \left(\frac{2(n+1) \pi x}{a}\right) d x-\int_{a / 2}^{a} q^{\prime}(a-x) \cos \left(\frac{2(n+1) \pi x}{a}\right) d x \\
& =\int_{0}^{a / 2} q^{\prime}(x) \cos \left(\frac{2(n+1) \pi x}{a}\right) d x+\int_{a / 2}^{0} q^{\prime}(u) \cos \left(\frac{2(n+1) \pi}{a}(a-u)\right) d u \\
& =\int_{0}^{a / 2} q^{\prime}(x) \cos \left(\frac{2(n+1) \pi x}{a}\right) d x+\int_{a / 2}^{0} q^{\prime}(u) \cos \left(\frac{2(n+1) \pi}{a} u\right) d u=0 .
\end{aligned}
$$

v) and vi) can be proved similarly to iv).

We obtain asymptotics approximations for symmetric single well potential as following:

Theorem 3.2. The eigenvalues $\lambda_{n}$ of (1.1)-(1.3) satisfy as $n \rightarrow \infty$,

i) if $a_{2}^{\prime} \neq 0$ and $b_{2}^{\prime} \neq 0$,

$$
\begin{aligned}
\lambda_{n}^{1 / 2} & =\frac{(n+1) \pi}{a}+\frac{1}{(n+1) \pi}\left\{\frac{a_{2}^{\prime} b_{1}^{\prime}-a_{1}^{\prime} b_{2}^{\prime}}{a_{2}^{\prime} b_{2}^{\prime}}-\frac{a}{2(n+1) \pi} \int_{0}^{a / 2} q^{\prime}(x)\left(\sin \frac{2(n+1) \pi x}{a}\right) d x\right. \\
& -\frac{a^{2}}{(n+1)^{2} \pi^{2}}\left[\frac{-3 b_{1}^{\prime} b_{2}^{\prime} b_{2}+3 b_{1}\left(b_{2}^{\prime}\right)^{2}+\left(b_{1}^{\prime}\right)^{3}}{3\left(b_{2}^{\prime}\right)^{3}}\right] \\
& \left.+\frac{a^{2}}{(n+1)^{2} \pi^{2}}\left[\frac{-3 a_{1}^{\prime} a_{2}^{\prime} a_{2}+3 a_{1}\left(a_{2}^{\prime}\right)^{2}+\left(a_{1}^{\prime}\right)^{3}}{3\left(a_{2}^{\prime}\right)^{3}}\right]\right\}+O\left(n^{-4} \eta(n)\right)+O\left(n^{-3} \eta^{2}(n)\right),
\end{aligned}
$$

ii) if $a_{2}^{\prime} \neq 0$ and $b_{2}^{\prime}=0$, 


$$
\begin{aligned}
\lambda_{n}^{1 / 2} & =\frac{(2 n+3) \pi}{2 a}+\frac{2}{(2 n+3) \pi}\left\{\frac{a_{2}^{\prime} b_{2}-a_{1}^{\prime} b_{1}^{\prime}}{a_{2}^{\prime} b_{1}^{\prime}}-\frac{a}{(2 n+3) \pi} \int_{0}^{a / 2} q^{\prime}(x)\left(\sin \frac{(2 n+3) \pi x}{a}\right) d x\right. \\
& +\frac{4 a^{2}}{(2 n+3)^{2} \pi^{2}}\left[\frac{3 b_{1}^{\prime} b_{1} b_{2}-b_{2}^{3}}{3\left(b_{1}^{\prime}\right)^{3}}\right]+\frac{4 a^{2}}{(2 n+3)^{2} \pi^{2}}\left[\frac{-3 a_{1}^{\prime} a_{2}^{\prime} a_{2}+3 a_{1}\left(a_{2}^{\prime}\right)^{2}+\left(a_{1}^{\prime}\right)^{3}}{3\left(a_{2}^{\prime}\right)^{3}}\right] \\
& \left.-\frac{4 a^{2}}{(2 n+3)^{2} \pi^{2}} \frac{a_{1}^{\prime}}{a_{2}^{\prime}} q(0)\right\}+O\left(n^{-4} \eta(n)\right)+O\left(n^{-3} \eta^{2}(n)\right) .
\end{aligned}
$$

Proof. $q(0)$ equals to $q(a)$ because of symmetric potential. By using this and Lemma 3.1 i), ii), iv), v) in Theorem 2.1, the theorem is proved.

Theorem 3.3. The eigenvalues $\lambda_{n}$ of (1.1)-(1.3) satisfy as $n \rightarrow \infty$,

i) if $a_{2}^{\prime}=0$ and $b_{2}^{\prime} \neq 0$,

$$
\begin{aligned}
\lambda_{n}^{1 / 2} & =\frac{(2 n+3) \pi}{2 a}+\frac{2}{(2 n+3) \pi}\left\{\frac{a_{1}^{\prime} b_{1}^{\prime}-a_{2} b_{2}^{\prime}}{a_{1}^{\prime} b_{2}^{\prime}}-\frac{a}{(2 n+3) \pi} \int_{0}^{a / 2} q^{\prime}(x)\left(\sin \frac{(2 n+3) \pi x}{a}\right) d x\right. \\
& -\frac{4 a^{2}}{(2 n+3)^{2} \pi^{2}}\left[\frac{3 b_{1}\left(b_{2}^{\prime}\right)^{2}-3 b_{1}^{\prime} b_{2}^{\prime} b_{2}+\left(b_{1}^{\prime}\right)^{3}}{3\left(b_{2}^{\prime}\right)^{3}}\right] \\
& \left.+\frac{4 a^{2}}{(2 n+3)^{2} \pi^{2}}\left[\frac{-3 a_{1}^{\prime} a_{1} a_{2}+a_{2}^{3}}{3\left(a_{1}^{\prime}\right)^{3}}\right]+\frac{4 a^{2}}{(2 n+3)^{2} \pi^{2}} \frac{a_{2}}{a_{1}^{\prime}} q(0)\right\}+O\left(n^{-4} \eta(n)\right)+O\left(n^{-3} \eta^{2}(n)\right),
\end{aligned}
$$

ii) if $a_{2}^{\prime}=0$ and $b_{2}^{\prime}=0$,

$$
\begin{aligned}
\lambda_{n}^{1 / 2} & =\frac{(n+2) \pi}{a}+\frac{1}{(n+2) \pi}\left\{\frac{a_{1}^{\prime} b_{2}-a_{2} b_{1}^{\prime}}{a_{1}^{\prime} b_{1}^{\prime}}+\frac{a}{2(n+2) \pi} \int_{0}^{a / 2} q^{\prime}(x)\left(\sin \frac{2(n+2) \pi x}{a}\right) d x\right. \\
& \left.-\frac{a^{2}}{(n+2)^{2} \pi^{2}}\left[\frac{3 b_{1}^{\prime} b_{1} b_{2}-b_{2}^{3}}{3\left(b_{1}^{\prime}\right)^{3}}\right]+\frac{a^{2}}{(n+2)^{2} \pi^{2}}\left[\frac{-3 a_{1}^{\prime} a_{1} a_{2}+a_{2}^{3}}{3\left(a_{1}^{\prime}\right)^{3}}\right]\right\}+O\left(n^{-4} \eta(n)\right)+O\left(n^{-3} \eta^{2}(n)\right) .
\end{aligned}
$$

Proof. The theorem follows from substitution of $q(0)=q(a)$ and Lemma 3.1 ii), iii), v), vi) into Theorem 2.2.

Example 3.4. Let us consider the equation

$$
y^{\prime \prime}(t)+\{\lambda-q(t)\} y(t)=0, \quad t \in[0, \pi],
$$

where $q(t)=\frac{1}{4}\left(t-\frac{\pi}{2}\right)^{4}+\frac{1}{2}\left(t-\frac{\pi}{2}\right)^{2}$. This equation is known anharmonic oscillator in quantum mechanics and $q(t)$ is symmetric single well. We assumed that $q(t)$ has mean value zero, so we should get $q(t)$ as following:

$$
q(t)=\frac{1}{4}\left(t-\frac{\pi}{2}\right)^{4}+\frac{1}{2}\left(t-\frac{\pi}{2}\right)^{2}-\frac{\pi^{2}}{24}-\frac{\pi^{4}}{320} .
$$

In this situation, by evaluating integral terms in Theorem 3.2 and Theorem 3.3, we find as $n \rightarrow \infty$ for $a_{2}^{\prime} \neq 0$ and $b_{2}^{\prime} \neq 0$, 


$$
\begin{aligned}
\lambda_{n}^{1 / 2} & =n+1+\frac{1}{(n+1) \pi}\left\{\frac{a_{2}^{\prime} b_{1}^{\prime}-a_{1}^{\prime} b_{2}^{\prime}}{a_{2}^{\prime} b_{2}^{\prime}}+\frac{\pi}{32(n+1)^{4}}\left[\left(\pi^{2}+4\right) n(n+2)+\pi^{2}-2\right]\right. \\
& \left.-\frac{1}{(n+1)^{2}}\left[\frac{-3 b_{1}^{\prime} b_{2}^{\prime} b_{2}+3 b_{1}\left(b_{2}^{\prime}\right)^{2}+\left(b_{1}^{\prime}\right)^{3}}{3\left(b_{2}^{\prime}\right)^{3}}\right]+\frac{1}{(n+1)^{2}}\left[\frac{-3 a_{1}^{\prime} a_{2}^{\prime} a_{2}+3 a_{1}\left(a_{2}^{\prime}\right)^{2}+\left(a_{1}^{\prime}\right)^{3}}{3\left(a_{2}^{\prime}\right)^{3}}\right]\right\} \\
& +O\left(n^{-4} \eta(n)\right)+O\left(n^{-3} \eta^{2}(n)\right),
\end{aligned}
$$

for $a_{2}^{\prime} \neq 0$ and $b_{2}^{\prime}=0$,

$$
\begin{aligned}
\lambda_{n}^{1 / 2} & =\frac{(2 n+3)}{2}+\frac{2}{(2 n+3) \pi}\left\{\frac{a_{2}^{\prime} b_{2}-a_{1}^{\prime} b_{1}^{\prime}}{a_{2}^{\prime} b_{1}^{\prime}}\right. \\
& -\frac{1}{8(2 n+3)^{5}}\left[8\left(4 n^{2}+12 n+3\right)(-1)^{n+1}-\pi(2 n+3)\left\{4\left(\pi^{2}+4\right) n(n+3)+9 \pi^{2}+12\right\}\right] \\
& +\frac{4}{(2 n+3)^{2}}\left[\frac{3 b_{1}^{\prime} b_{1} b_{2}-b_{2}^{3}}{3\left(b_{1}^{\prime}\right)^{3}}\right]+\frac{4}{(2 n+3)^{2}}\left[\frac{-3 a_{1}^{\prime} a_{2}^{\prime} a_{2}+3 a_{1}\left(a_{2}^{\prime}\right)^{2}+\left(a_{1}^{\prime}\right)^{3}}{3\left(a_{2}^{\prime}\right)^{3}}\right] \\
& \left.-\frac{4}{(2 n+3)^{2}} \frac{a_{1}^{\prime}}{a_{2}^{\prime}}\left[\frac{\pi^{4}}{80}+\frac{\pi^{2}}{12}\right]\right\}+O\left(n^{-4} \eta(n)\right)+O\left(n^{-3} \eta^{2}(n)\right),
\end{aligned}
$$

for $a_{2}^{\prime}=0$ and $b_{2}^{\prime} \neq 0$,

$$
\begin{aligned}
\lambda_{n}^{1 / 2} & =\frac{(2 n+3)}{2}+\frac{2}{(2 n+3) \pi}\left\{\frac{a_{1}^{\prime} b_{1}^{\prime}-a_{2} b_{2}^{\prime}}{a_{1}^{\prime} b_{2}^{\prime}}\right. \\
& -\frac{1}{8(2 n+3)^{5}}\left[8\left(4 n^{2}+12 n+3\right)(-1)^{n+1}-\pi(2 n+3)\left\{4\left(\pi^{2}+4\right) n(n+3)+9 \pi^{2}+12\right\}\right] \\
& -\frac{4}{(2 n+3)^{2}}\left[\frac{3 b_{1}\left(b_{2}^{\prime}\right)^{2}-3 b_{1}^{\prime} b_{2}^{\prime} b_{2}+\left(b_{1}^{\prime}\right)^{3}}{3\left(b_{2}^{\prime}\right)^{3}}\right]+\frac{4}{(2 n+3)^{2}}\left[\frac{-3 a_{1}^{\prime} a_{1} a_{2}+a_{2}^{3}}{3\left(a_{1}^{\prime}\right)^{3}}\right] \\
& \left.+\frac{4}{(2 n+3)^{2}} \frac{a_{2}}{a_{1}^{\prime}}\left[\frac{\pi^{4}}{80}+\frac{\pi^{2}}{12}\right]\right\}+O\left(n^{-4} \eta(n)\right)+O\left(n^{-3} \eta^{2}(n)\right),
\end{aligned}
$$

for $a_{2}^{\prime}=0$ and $b_{2}^{\prime}=0$,

$$
\begin{aligned}
\lambda_{n}^{1 / 2} & =n+2+\frac{1}{(n+2) \pi}\left\{\frac{a_{1}^{\prime} b_{2}-a_{2} b_{1}^{\prime}}{a_{1}^{\prime} b_{1}^{\prime}}-\frac{\pi}{32(n+2)^{4}}\left[\left(\pi^{2}+4\right) n(n+4)+4 \pi^{2}+10\right]\right. \\
& \left.-\frac{1}{(n+2)^{2}}\left[\frac{3 b_{1}^{\prime} b_{1} b_{2}-b_{2}^{3}}{3\left(b_{1}^{\prime}\right)^{3}}\right]+\frac{1}{(n+2)^{2}}\left[\frac{-3 a_{1}^{\prime} a_{1} a_{2}+a_{2}^{3}}{3\left(a_{1}^{\prime}\right)^{3}}\right]\right\}+O\left(n^{-4} \eta(n)\right)+O\left(n^{-3} \eta^{2}(n)\right) .
\end{aligned}
$$

\section{ACKNOWLEDGEMENT}

The author is grateful to Prof. Dr. Haskız COŞKUN for supervisor.

\section{CONFLICTS OF INTEREST}

The author declares that there are no conflicts of interest regarding the publication of this article. 


\section{REFERENCES}

[1] Andrews, B., Clutterbuck, J., Hauer, D., The fundamental gap for a one-dimensional Schrödinger operator with Robin boundary conditions, arXiv:2002.06900 [math.CA], (2020).

[2] Ashbaugh M. S., Benguria, R., Optimal lower bound for the gap between the first two eigenvalues of one-dimensional Schrödinger operators with symmetric single-well potentials and related results, Proceedings of the American Mathematical Society, 105(1989), 419-424.

[3] Ashbaugh, M. S., Kielty, D., Spectral gaps of 1-D Robin Schödinger operators with single well potentials, Journal of Mathematical Physics, 61 (9)(2020), 091507.

[4] Başkaya, E., Asymptotics of eigenvalues for Sturm-Liouville problem with eigenparameter-dependent boundary conditions, New Trends in Mathematical Sciences, 6(2)(2018), 247-257.

[5] Capała, K., Dybiec, B., Multimodal stationary states in symmetric single-well potentials driven by Cauchy noise, Journal of Statistical Mechanics: Theory and Experiment, 2019(2019), 033206.

[6] Chen, W.-C., Cheng, Y.-H., Remarks on the one-dimensional sloshing problem involving the p-Laplacian operator, Turkish Journal of Mathematics, 44(2020), 1376-1387.

[7] Chen, D.- Y., Huang, M.- J., Comparison theorems for the eigenvalue gap of Schrödinger operators on the real line, Annales Henri Poincaré, 13(2011), 85-101.

[8] Ciesla, M., Capała, K., Dybiec, B., Multimodal stationary states under Cauchy noise, Physical Review E, 99(2019), 052118.

[9] Coşkun, H., Başkaya, E., Asymptotics of eigenvalues of regular Sturm-Liouville problems with eigenvalue parameter in the boundary condition for integrable potential, Mathematica Scandinavica, 107(2017), 209-223.

[10] Coşkun, H., Bayram, N., Asymptotics of eigenvalues for regular Sturm-Liouville problems with eigenvalue parameter in the boundary condition, Journal of Mathematical Analysis and Applications, 306(2)(2005), 548-566.

[11] Coşkun, H., Kabataş, A., Asymptotic approximations of eigenfunctions for regular Sturm-Liouville problems with eigenvalue parameter in the boundary condition for integrable potential, Mathematica Scandinavica, 113(1)(2013), 143-160.

[12] Coşkun, H., Kabataş, A., Green's function of regular Sturm-Liouville problem having eigenparameter in one boundary condition, Turkish Journal of Mathematics and Computer Science, 4(2016), 1-9.

[13] Coşkun, H., Kabataş, A., Başkaya, E., On Green's function for boundary value problem with eigenvalue dependent quadratic boundary condition, Boundary Value Problems, 71(2017).

[14] Coşkun, H., Başkaya, E., Kabataş, A., Instability intervals for Hill's equation with symmetric single well potential, Ukrainian Mathematical Journal, 71(6)(2019), 977-983.

[15] Fulton, C., Two point boundary value problems with eigenvalue parameter contained in the boundary conditions, Proceedings of the Royal Society of Edinburgh, 77A(1977), 293-308.

[16] Fulton, C., Singular eigenvalue problems with eigenvalue parameter contained in the boundary conditions, Proceedings of the Royal Society of Edinburgh, 87A(1980), 1-34.

[17] Guliyev, N. J., Schrödinger operators with distributional potentials and boundary conditions dependent on the eigenvalue parameter, Journal of Mathematical Physics, 60(6)(2019), 063501.

[18] Guliyev, N. J., Inverse square singularities and eigenparameter dependent boundary conditions are two sides of the same coin, arXiv:2001.00061 [math-ph], 2019.

[19] Guliyev, N. J., Essentially isospectral transformations and their applications, Annali di Matematica Pura ed Applicata, 199(2020), $1621-1648$.

[20] Guliyev, N. J., On two-spectra inverse problems, Proceedings of the American Mathematical Society, 148(2020), 4491-4502.

[21] Haaser, N. B., Sullivian, J. A., Real Analysis, Van Nostrand Reinhold Company, New York, 1991.

[22] Hinton, D. B., Eigenfunction expansions for a singular eigenvalue problem with eigenparameter in the boundary condition, SIAM Journal on Mathematical Analysis, 12(1981), 572-584.

[23] Horvath, M., On the first two eigenvalues of Sturm-Liouville Operators, Proceedings of the American Mathematical Society, 131(4)(2002), $1215-1224$.

[24] Huang, M. J., The first instability interval for Hill equations with symmetric single well potentials, Proceedings of the American Mathematical Society, 125(1997), 775-778.

[25] Huang, M. J., Tsai, T. M., The eigenvalue gap for one-dimensional Schrodinger operators with symmetric potentials, Proceedings of the Royal Society of Edinburgh Section A, 139(2009), 359-366.

[26] Kerner, J., Täufer, M., On the spectral gap of one-dimensional Schrödinger operators on large intervals, arXiv:2012.09060 [math.SP], 2020.

[27] Mandrysz, M., Dybiec, B., Energetics of single-well undamped stochastic oscillators, Physical Review E, 99(1)(2019), 012125.

[28] Messori, C., Deep into the Water: Exploring the Hydro-Electromagnetic and QuantumElectrodynamic Properties of Interfacial Water in Living Systems, Open Access Library Journal, 6 (2019), e5435. 